\title{
THE ITERATED PROJECTION SOLUTION FOR THE FREDHOLM INTEGRAL EQUATION OF SECOND KIND
}

\author{
FRANÇOISE CHATELIN and RACHID LEBBAR
}

(Received 21 August 1980)

(Revised 11 November 1980)

\begin{abstract}
We are concerned with the solution of the second kind Fredholm equation (and eigenvalue problem) by a projection method, where the projection is either an orthogonal projection on a set of piecewise polynomials or an interpolatory projection at the Gauss points of subintervals.

We study these cases of superconvergence of the Sloan iterated solution: global superconvergence for a smooth kernel, and superconvergence at the partition points for a kernel of "Green's function" type. The mathematical analysis applies for the solution of the inhomogeneous equation as well as for an eigenvector.
\end{abstract}

\section{Introduction}

We consider some projection methods for the solution of second kind integral equations of the form

$$
(T x)(s)-z x(s)=f(s), \quad 0 \leqslant s<1,
$$

where $T$ is the operator defined by

$$
x(s) \mapsto \int_{0}^{1} k(s, t) x(t) d t, \quad 0<s<1 .
$$

Along with (1), we consider the eigenvalue problem

$$
(T \phi)(s)=\lambda \phi(s), \quad 0<s<1, \phi \neq 0 .
$$

(1) and (2) are regarded as equations in an appropriate subspace $X$ of the complex Banach space $L^{\infty}(0,1)$ with the norm $\|\cdot\|_{\infty} . T$ is supposed to be 
compact and $z \in \rho(T)$, the resolvent set of $T$, so that $(T-z)^{-1}$ is bounded with domain $X$. Let $X_{n}$ be a finite dimensional subspace of $X$ and let $\Pi_{n}$ be a projection onto $X_{n}$. Then the projection method consists in approximating (1) and (2) respectively by

$$
\begin{gathered}
\left(\Pi_{n} T-z\right) x_{n}=\Pi_{n} f, \quad x_{n} \in X_{n}, \\
\Pi_{n} T \phi_{n}=\lambda_{n} \phi_{n}, \quad 0 \neq \phi_{n} \in X_{n},
\end{gathered}
$$

where $x_{n}$ (resp. $\phi_{n}$ ) is the projection solution (iesp. eigenvectur), corresponding to the approximation $T_{n}^{P}=\Pi_{n} T$ of $T$ ( $P$ for projection).

Given a projection $\Delta=\left\{t_{i}\right\}_{0}^{n}$ of $[0,1], t_{0}=0, t_{n}=1$, let $X_{n}$ be a space $S_{\Delta}$ of piecewise polynomials of degree $\left\langle r\right.$ on each subinterval $\Delta_{i}=\left[t_{i-1}, t_{i}\right]$, $i=1, \ldots, n$. We set $h=\max _{1<i<n}\left(t_{i}-t_{i-1}\right)$. We shall consider two types of projection methods:

(a) $\Pi_{n}$ is the orthogonal projection (in $L^{2}(0,1)$ ) on $S_{\Delta}$,

(b) $\Pi_{n}$ is an interpolatory projection defined so that $\Pi_{n} x$ is the piecewise polynomial of degree $\leqslant r$ which interpolates $x$ at $r+1$ points $\left\{\tau_{j}^{i}\right\}_{j=1}^{r+1}$, on each $\Delta_{i}, i=1, \ldots, n$.

Case (a) corresponds to a Galerkin method, and case (b) to a collocation method at the collocation points $\left\{\tau_{j}^{i}\right\}$.

If $z \neq 0$ (resp. $\lambda_{n} \neq 0$ ) we consider the iterated projection solution $\tilde{x}_{n}$ (resp. eigenvector $\tilde{\phi}_{n}$ ) introduced by Sloan [13], [14] and given by the formulae:

$$
\tilde{x}_{n}=\frac{1}{z}\left(T x_{n}-f\right), \quad \tilde{\phi}_{n}=\frac{1}{\lambda_{n}} T \phi_{n},
$$

where $\tilde{x}_{n}$ and $\tilde{\phi}_{n}$ are solutions of the equations

$$
\left(T \Pi_{n}-z\right) \tilde{x}_{n}=f \text {, }
$$

and

$$
T \Pi_{n} \tilde{\phi}_{n}=\lambda_{n} \tilde{\phi}_{n}
$$

corresponding to the approximation $T_{n}^{S}=T \Pi_{n}$ of $T$ ( $S$ for Sloan). Now $\Pi_{n} \tilde{x}_{n}=x_{n}$ and $\Pi_{n} \tilde{\phi}_{n}=\phi_{n}$, so that in case (b), the iterated solutions and the solutions themselves agree at the collocation points.

If $k$ and $f$ are smooth enough, it is known that $\left\|x_{n}-x\right\|_{\infty}=O\left(h^{r+1}\right)$, while $\left\|\tilde{x}_{n}-x\right\|_{\infty}=O\left(h^{2 r+2}\right)$ for case (b) for example, provided that the $\left\{\tau_{j}^{i}\right\}$ are the $r+1$ Gauss points on $\Delta_{i}, i=1, \ldots, n$. The optimal rate of convergence, relative to $S_{\Delta}$, which is $\inf _{y \in S_{\Delta}}\|x-y\|_{\infty}=O\left(h^{r+1}\right)$, is then overshot by $\tilde{x}_{n} \notin S_{\Delta}$, when $k$ and $f$ are smooth. Such fast convergence is often called superconvergence.

When $k$ is the Green's function of an ordinary differential equation (o.d.e.) of order $p$ with smooth coefficients, $\tilde{x}_{n}$ is still superconvergent at the partition points $\left\{t_{i}\right\}_{0}^{n}$, but not globally: the global rate of convergence is now $O\left(h^{r+1+p}\right)$. 
Similar results hold for $\tilde{\phi}_{n}$. This problem is studied for the equation (1) and the Galerkin method in Chandler's thesis [6]. The collocation method for a non linear o.d.e. has been looked at by de Boor-Swartz (see [1] for the solution of (1), and [2], [3] for the linear eigenvalue problem (2)), where $T$ is the associated differential operator. In de Boor-Swartz [4] the "essential" least squares method (or local moment method) for an o.d.e. is also studied.

We present in this paper an analysis of the convergence rates which is a blend of the techniques of Chandler and of de Boor-Swartz. It applies for the iterated solution $\tilde{x}_{n}$ as well as for the iterated eigenvector $\tilde{\phi}_{n}$ (the result seems to be new for the eigenvector in the most general case). It is . ased on a study of the error at the point $t$ of $[0,1]$ in terms of the scalar product $\left\langle l_{t},\left(1-\Pi_{n}\right) \tilde{x}_{n}\right\rangle$ (resp. $\left.\left\langle l_{t}^{\prime},\left(1-\Pi_{n}\right) \tilde{\phi}_{n}\right\rangle\right)$ where $l_{t}$ (resp. $\left.l_{t}^{\prime}\right)$ is a function having the same smoothness properties as $k_{t}(\cdot):=k(t, \cdot)$, and where $\langle f, g\rangle=\int_{0}^{1} f \bar{g}$.

In case (a), we use the orthogonality of $\Pi_{n}$ :

$$
\left\langle l_{t},\left(1-\Pi_{n}\right) \tilde{x}_{n}\right\rangle=\left\langle\left(1-\Pi_{n}\right) l_{t},\left(1-\Pi_{n}\right) \tilde{x}_{n}\right\rangle .
$$

In case (b) we use firstly that the function $\left(1-\Pi_{n}\right) \tilde{x}_{n}$ vanishes at the collocation points $\tau_{j}^{i}$, and secondly that the $\left\{\tau_{j}^{i}\right\}$ being the $r+1$ Gauss points in $\Delta_{i}$, then $\int_{\Delta_{i}} p(s) \Pi_{j=1}^{r+1}\left(s-\tau_{j}^{i}\right) d s=0$ for all polynomials $p$ of degree $<r$.

The superconvergence in case (a) is proved under the assumption that $\Delta$ is quasi-uniform. In case (b), $\Delta$ is arbitrary but more smoothness properties are required for $k$ and $f$.

\section{The setting of the problem}

\subsection{Piecewise continuous functions}

Let be given $\Delta=\left\{t_{i}\right\}_{0}^{n}$, a strict partition of $[0,1], 0=t_{0}<t_{1}<\cdots<t_{n}=1$. It is quasi-uniform if there exists $\sigma \geqslant 0: \max \left(t_{i}-t_{i-1}\right) / \min \left(t_{i}-t_{i-1}\right) \leqslant \sigma$ for $n=1,2, \ldots$ Then $n h \leqslant \sigma . \Delta_{i}:=\left[t_{i-1}, t_{i}\right], i=1,2, \ldots, n$. We define $C_{\Delta}:=\prod_{i=1}^{n} C_{\left(\Delta_{1}\right)}: f \in C_{\Delta}$ consists of $n$ components $f_{i} \in C_{\left(\Delta_{i}\right)}, f$ is a piecewise continuous function having (possibly) different left and right values at the partition points $t_{i}$. With the norm $\|\cdot\|_{\Delta}$ defined by $\|f\|_{\Delta}=\max _{i=1, \ldots, n}\left\|f_{i}\right\|_{\infty}$, $C_{\Delta}$ is a Banach space. $C_{\Delta} \subset L^{\infty}(0,1)$ by $\|f\|_{\Delta}<\|f\|_{\infty}$ and if $f$ is continuous on $[0,1]$, then $\|f\|_{\infty}=\|f\|_{\Delta}$. We define, more generally, $C_{\Delta}^{l}$ for positive integer $l$ by $C_{\Delta}^{\prime}=\Pi_{i=1}^{n} C_{\left(\Delta_{l}\right)}^{l}$ where $f_{i} \in C_{(\Delta,)}^{\prime}$ iff its $/$ th derivative $f_{i}^{(l)}$ is continuous on $\Delta_{i}$. Clearly $S_{\Delta} \subset C_{\Delta}$ and the projection $\Pi_{n}$ is defined $C_{\Delta} \rightarrow S_{\Delta}$ with $f=\left(f_{1}, \ldots, f_{n}\right)$ $\mapsto \Pi_{n} f=\left(\Pi f_{1}, \ldots, \Pi f_{n}\right)$, where $\Pi f_{1}$ is the projection of $f_{i} \in C_{\left(\Delta_{l}\right)}$ on the polynomials of degree $<r$ on $\Delta_{i}$. 


\subsection{Spectral definitions}

$T$ is supposed to be compact in the complex Banach space $X=C_{\Delta} \cdot \mathcal{L}(X)$ is the algebra of bounded operators on $X$. The resolvent set of $T$ is $\rho(T)=\{z \in \mathbf{C}$; $\left.(T-z)^{-1} \in \mathcal{L}(X)\right\}$ where $z$ stands for $z 1$. For $z$ in $\rho(T), R(z)=(T-z)^{-1}$ is the resolvent of $T$ and $T R(z)=R(z) T$. The unique solution of (1) is then $x=R(z) f$.

Let $\lambda \neq 0$ be an isolated eigenvalue of $T$ with algebraic (resp. geometric) multiplicity $m$ (resp. $g$ ), and ascent $\mu, 1<\mu<m, 1<g<m$. The associated eigenspace is $E=\operatorname{ker}(T-\lambda)$, the null space of $T-\lambda$ so $\operatorname{dim} E=g$; the invariant subspace is

$$
M=\operatorname{ker}(T-\lambda)^{m}, \quad \operatorname{dim} M=m, \quad \text { and } \quad \operatorname{ker}(T-\lambda)^{\mu} \equiv \operatorname{ker}(T-\lambda)^{m} .
$$

Let $\Gamma$ be a Jordan curve in $\rho(T)$, around $\lambda$, which contains neither 0 nor any other eigenvalue of $T . P:=-1 / 2 i \pi \int_{\Gamma} R(z) d z$ is the spectral projection associated with $\lambda, M=P X$. Let $T_{n}$ be a sequence of operators in $\mathcal{E}(X)$ such that $T_{n}$ converge to $T$ pointwise. $T_{n}$ will be either $T_{n}^{P}=\Pi_{n} T$ or $T_{n}^{S}=T \Pi_{n}$. If $\Gamma \subset$ $\rho\left(T_{n}\right)$, we may define for $T_{n}$ the resolvent $R_{n}(z)$ for $z \in \Gamma$ and the spectral projection $P_{n}:=-1 / 2 i \pi \int_{\Gamma} R_{n}(z) d z$. If $T_{n}$ is strongly stable inside $\Gamma$ (Chatelin [8], [9]), there are, for $n$ large enough, exactly $m$ eigenvalues $\left\{\lambda_{i n}\right\}_{i=1}^{m}$ of $T_{n}$ inside $\Gamma$ (counting their algebraic multiplicities), $\hat{\lambda}_{n}$ is their arithmetic mean, and $\lambda_{n}$ is any one of them.

For the projections $\Pi_{n}$ under consideration, both $T_{n}^{P}$ and $T_{n}^{S}$ are strongly stable around any non-zero eigenvalue of $T$ (Chatelin [7], [9]). The solution $x_{n}$ of (3) is such that $x_{n}=R_{n}^{P}(z) \Pi_{n} f$, and $\tilde{x}_{n}=R_{n}^{S}(z) f$. Similarly $\phi_{n}$ is an eigenvector of $T_{n}^{P}$ and $\tilde{\phi}_{n}$ of $T_{n}^{S}$, associated with the same eigenvalue $\lambda_{n}$.

\subsection{The errors $x_{n}-x, \phi_{n}-P \phi_{n}, \tilde{x}_{n}-x, \tilde{\phi}_{n}-P \tilde{\phi}_{n}$ and $\lambda-\hat{\lambda}_{n}$}

$C$ is a generic constant, which may depend on $r$ and $\sigma$, but is otherwise independent of $\Delta$.

\subsubsection{The projection method}

We recall the following equality:

$$
x_{n}-x=z R_{n}^{P}(z)\left(1-\Pi_{n}\right) x, \text { then }\left\|x-x_{n}\right\|_{\infty} \leqslant C\left\|\left(1-\Pi_{n}\right) x\right\|_{\infty} .
$$

As for the resolvents,

$$
\left(R_{n}^{P}(z)-R(z)\right) \phi_{n}=R(z)\left(T-T_{n}^{P}\right) R_{n}^{P}(z) \phi_{n}=\frac{R(z)}{\lambda_{n}-z}\left(1-\Pi_{n}\right) T \phi_{n},
$$


because $R_{n}^{P}(z) \phi_{n}=\phi_{n} / \lambda_{n}-z$. To integrate on $\Gamma$, we distinguish whether $\lambda_{n}=\lambda$ or not. If $\lambda_{n}=\lambda$, then $-1 / 2 i \pi \int_{\Gamma}(R(z) / \lambda-z) d z=S=\lim _{z \rightarrow \lambda} R(z)(1-P)$; $S$ is the reduced resolvent with respect to $\lambda$. If $\lambda_{n} \neq \lambda, R(z)-R\left(\lambda_{n}\right)=$ $\left(z-\lambda_{n}\right) R\left(\lambda_{n}\right) R(z)$, and

$$
\frac{-1}{2 i \pi} \int_{\Gamma} \frac{R(z)}{\lambda_{n}-z} d z=R\left(\lambda_{n}\right)\left[\frac{-1}{2 i \pi} \int_{\Gamma} \frac{d z}{\lambda_{n}-z}+\frac{1}{2 i \pi} \int_{\Gamma} R(z) d z\right]=R\left(\lambda_{n}\right)(1-P) .
$$

$\lambda$ is the only pole of $R(z)$ inside $\Gamma, R\left(\lambda_{n}\right)(1-P)$ is well defined and when $n \rightarrow \infty, \lambda_{n} \rightarrow \lambda, R\left(\lambda_{n}\right)(1-P) \rightarrow S . R\left(\lambda_{n}\right)(1-P)$ is then uniformly bounded in $n$, for $n$ large enough. To have a unique formula for the cases $\lambda_{n}=\lambda$ and $\lambda_{n} \neq \lambda$, we set $R(\lambda)(1-P)=S$.

By integration in $z$ on $\Gamma$, we get $\phi_{n}-P \phi_{n}=R\left(\lambda_{n}\right)(1-P)\left(1-\Pi_{n}\right) T \phi_{n}$, and $\operatorname{dist}\left(\phi_{n}, M\right)=\inf _{\phi \in M}\left\|\phi_{n}-\phi\right\|_{\infty}<\left\|\phi_{n}-P \phi_{n}\right\|_{\infty}<C\left\|\left(1-\Pi_{n}\right) T \phi_{n}\right\|_{\infty}$.

\subsubsection{The Sloan method}

1) $\tilde{x}_{n}-x=\left(R_{n}^{S}(z)-R(z)\right) f=R(z)\left(T-T_{n}^{S}\right) R_{n}^{S} f=R(z) T\left(1-\Pi_{n}\right) \tilde{x}_{n}=$ $\operatorname{TR}(z)\left(1-\Pi_{n}\right) \tilde{x}_{n}$.

Then for any fixed $t$ in $[0,1]$, and any fixed $z$ in $\rho(T)$,

$$
\begin{aligned}
\left(\tilde{x}_{n}-x\right)(t) & =\int_{0}^{1} k(t, s)\left[R(z)\left(1-\Pi_{n}\right) \tilde{x}_{n}\right](s) d s \\
& =\left\langle k_{t}, R(z)\left(1-\Pi_{n}\right) \tilde{x}_{n}\right\rangle \\
& =\left\langle(R(z))^{*} k_{t},\left(1-\Pi_{n}\right) \tilde{x}_{n}\right\rangle=\left\langle l,\left(1-\Pi_{n}\right) \tilde{x}_{n}\right\rangle .
\end{aligned}
$$

Because $R^{*}(z):=(R(z))^{*}=\left(T^{*}-\bar{z}\right)^{-1}, l_{t}:=R^{*}(z) k_{t}$ is the solution of $\left(T^{*}-\bar{z}\right) l_{t}=k_{t}$; the solution $l_{t}$ (which depends on $z$ ) is unique since $z \in \rho(T) \Leftrightarrow$ $\bar{z} \in \rho\left(T^{*}\right)$.

2) Similarly

$$
\left(R_{n}^{S}(z)-R(z)\right) \tilde{\phi}_{n}=R(z)\left(T-T_{n}^{S}\right) R_{n}^{S}(z) \tilde{\phi}_{n}=\frac{R(z)}{\lambda_{n}-z} T\left(1-\Pi_{n}\right) \tilde{\phi}_{n}
$$

By integration on $\Gamma$, we get for any fixed $t$ on $[0,1]$

$$
\begin{aligned}
\tilde{\phi}_{n}(t)-\left(P \tilde{\phi}_{n}\right)(t) & =\left[T\left(\frac{-1}{2 i \pi} \int_{\Gamma} \frac{R(z)}{\lambda_{n}-z} d z\right)\left(1-\Pi_{n}\right) \tilde{\phi}_{n}\right](t) \\
& =\left[T R\left(\lambda_{n}\right)(1-P)\left(1-\Pi_{n}\right) \tilde{\phi}_{n}\right](t) .
\end{aligned}
$$

We define $l_{t}^{\prime}:=R^{*}\left(\lambda_{n}\right)\left(1-P^{*}\right) k_{t}$, that is $l_{l}^{\prime}$ is the unique solution of $\left(T^{*}-\bar{\lambda}_{n}\right) l_{t}^{\prime}$ $=\left(1-P^{*}\right) k_{t}$. We define accordingly $R^{*}(\lambda)\left(1-P^{*}\right):=S^{*}$. Then $\tilde{\phi}_{n}(t)-$ $\left(P \tilde{\phi}_{n}\right)(t)=\left\langle l_{t}^{\prime},\left(1-\Pi_{n}\right) \tilde{\phi}_{n}\right\rangle$. We have just proved that the error $\left(x-\tilde{x}_{n}\right)(t)$ (resp. $\left.\left(\tilde{\phi}_{n}-P \tilde{\phi}_{n}\right)(t)\right)$ at $t \in[0,1]$ can be expressed in terms of the scalar product $\left\langle l_{l},\left(1-\Pi_{n}\right) \tilde{x}_{n}\right\rangle\left(\operatorname{resp} .\left\langle l_{l}^{\prime},\left(1-\Pi_{n}\right) \tilde{\phi}_{n}\right\rangle\right)$. 
ReMark. Another way to bound

$$
\left(\tilde{\phi}_{n}-P \tilde{\phi}_{n}\right)(t)=\left[T\left(-1 / 2 i \pi \int_{\Gamma}\left(R(z) / \lambda_{n}-z\right) d z\right)\left(1-\Pi_{n}\right) \tilde{\phi}_{n}\right](t)
$$

is the following (Lebbar [10]). Let $\Gamma^{\prime}$ be the circle centered at $\lambda_{n}$, with radius $r$, containing $\lambda$ and contained in $\Gamma$ (for $n$ large enough, there exists such a circle). We set $z=\lambda_{n}+r e^{i \theta}, 0 \leqslant \theta<2 \pi$, for $z \in \Gamma^{\prime}$.

$$
\begin{aligned}
\frac{-1}{2 i \pi} \int_{\Gamma} \frac{R(z)}{\lambda_{n}-z} d z & =\frac{-1}{2 i \pi} \int_{\Gamma^{\prime}} \frac{R(z)}{\lambda_{n}-z} d z \\
& =\frac{-1}{2 i \pi} \int_{0}^{2 \pi} \frac{R\left(\lambda_{n}+r e^{i \theta}\right)}{-r e^{i \theta}} r i e^{i \theta} d \theta .
\end{aligned}
$$

Then

$$
\begin{aligned}
\left|\left(\tilde{\phi}_{n}-P \tilde{\phi}_{n}\right)(t)\right| & =\frac{1}{2 \pi}\left|\int_{0}^{2 \pi}\left[\operatorname{TR}\left(\lambda_{n}+r e^{i \theta}\right)\left(1-\Pi_{n}\right) \tilde{\phi}_{n}\right](t) d \theta\right| \\
& \leqslant \sup _{0<\theta<2 \pi}\left|\left[\operatorname{TR}\left(\lambda_{n}+r e^{i \theta}\right)\left(1-\Pi_{n}\right) \tilde{\phi}_{n}\right](t)\right| \\
& =\sup _{z \in \Gamma^{\prime}}\left|\left[\operatorname{TR}(z)\left(1-\Pi_{n}\right) \tilde{\phi}_{n}\right](t)\right| .
\end{aligned}
$$

For $z \in \Gamma^{\prime}$, we define $l_{t}(z):=R^{*}(z) k_{t}$. Then

$$
\left|\left(\tilde{\phi}_{n}-P \tilde{\phi}_{n}\right)(t)\right| \leqslant \sup _{z \in \Gamma^{\prime}}\left|\left\langle l_{t}(z),\left(1-\Pi_{n}\right) \tilde{\phi}_{n}\right\rangle\right| \text {. }
$$

As for the global bounds on $[0,1]$, they are easy to get:

$$
r \tilde{x}_{n}-x=R(z) T\left(1-\Pi_{n}\right) \tilde{x}_{n}
$$

implies $\left\|\tilde{x}_{n}-x\right\|_{\infty} \leqslant C\left\|T\left(1-\Pi_{n}\right) \tilde{x}_{n}\right\|_{\infty}$, and

$$
\left\|T\left(1-\Pi_{n}\right) \tilde{x}_{n}\right\|_{\infty}=\sup _{t \in[0,1]}\left|\left\langle k_{t},\left(1-\Pi_{n}\right) \tilde{x}_{n}\right\rangle\right| \text {. }
$$

$\tilde{\phi}_{n}-P \tilde{\phi}_{n}=R\left(\lambda_{n}\right)(1-P) T\left(1-\Pi_{n}\right) \tilde{\phi}_{n}$ implies

$$
\operatorname{dist}\left(\tilde{\phi}_{n}, M\right):=\inf _{\phi \in M}\left\|\tilde{\phi}_{n}-\phi\right\|_{\infty} \leqslant\left\|\tilde{\phi}_{n}-P \tilde{\phi}_{n}\right\|_{\infty} \leqslant C\left\|T\left(1-\Pi_{n}\right) \tilde{\phi}_{n}\right\|_{\infty},
$$

and

$$
\left\|T\left(1-\Pi_{n}\right) \tilde{\phi}_{n}\right\|_{\infty}=\sup _{t \in[0,1]}\left|\left\langle k_{t},\left(1-\Pi_{n}\right) \tilde{\phi}_{n}\right\rangle\right|
$$

3) Now we set $M_{n}:=P_{n} X$. For $n$ large enough, $P_{P_{M_{n}}}$ has a bounded inverse and $m\left(\lambda-\hat{\lambda}_{n}\right)=\sum_{i-1}^{m}\left\langle x_{i}^{*},\left(1-\Pi_{n}\right) T\left(P_{I_{M}}\right)^{-1} x_{i}\right\rangle$ where $\left\{x_{i}\right\}_{1}^{m}$ (resp. $\left.\left\{x_{i}^{*}\right\}_{1}^{m}\right)$ is a basis of $M$ (resp. the adjoint basis of $M^{*}$ ) (see de Boor-Swartz [2]). The error $\lambda-\hat{\lambda}_{n}$ is then of the same type as the errors $\left(\tilde{x}_{n}-x\right)(t)$ and $\left(\tilde{\phi}_{n}-P \tilde{\phi}_{n}\right)(t)$.

4) Let $Q$ be the eigenprojection on $E=\operatorname{ker}(T-\lambda)$, along a supplementary subspace $F$. 


$$
\tilde{\phi}_{n}-Q \tilde{\phi}_{n}=[(T-\lambda)(1-Q)]^{-1}(T-\lambda)(1-Q) \tilde{\phi}_{n},
$$

and

$$
(T-\lambda)(1-Q) \tilde{\phi}_{n}=(T-\lambda) \tilde{\phi}_{n}=T\left(1-\Pi_{n}\right) \tilde{\phi}_{n}+\left(\lambda_{n}-\lambda\right) \tilde{\phi}_{n} .
$$

Therefore

$$
\begin{aligned}
\operatorname{dist}\left(\tilde{\phi}_{n}, E\right) & =\inf _{\phi \in E}\left\|\tilde{\phi}_{n}-\phi\right\|_{\infty} \leqslant\left\|\tilde{\phi}_{n}-Q \tilde{\phi}_{n}\right\|_{\infty} \\
& \leqslant C\left(\left\|T\left(1-\Pi_{n}\right) \tilde{\phi}_{n}\right\|_{\infty}+\left|\lambda_{n}-\lambda\right|\right) .
\end{aligned}
$$

Note that this method does not provide a pointwise estimate for $\tilde{\phi}_{n}-Q \tilde{\phi}_{n}$. This is due to the fact that, unlike the spectral projection $P$, the eigenprojection $Q$ has no expression in terms of the resolvent.

\section{Two basic results}

We shall be concerned with two types of continuous kernels $k$ that we define now.

i) $k$ is smooth (of order $l \geqslant 0$ ) if $k \in C_{\Delta}^{l}\left([0,1] \times[0,1]\right.$ ), that is $k_{i j} \in C_{\left(\Delta_{i} \times \Delta_{j}\right)}^{l}$ ), for $1 \leqslant i, j \leqslant n$, and $k$ is continuous on $[0,1] \times[0,1]$.

ii) $k$ is a Green's kernel (of order $l \geqslant 1$, and continuity $\delta, 0 \leqslant \delta<l$ ) if

$$
k(t, s)= \begin{cases}k_{1}(t, s) & \text { for } t \geqslant s, \\ k_{2}(t, s) & \text { for } t<s,\end{cases}
$$

is such that

$$
\begin{gathered}
k_{1} \in C^{\prime}(\{0 \leqslant s \leqslant t \leqslant 1\}), \\
k_{2} \in C^{\prime}(\{0<t<s \leqslant 1\}), \\
k \in C^{\delta}([0,1] \times[0,1]) .
\end{gathered}
$$

An obvious example of case ii) is the Green's function of an o.d.e. of order $\delta+2$.

For any $z$ in $\rho(T)$, we consider the solution $x=R(z) f$ of (1), along with $\tilde{x}_{n}$ and $\tilde{\phi}_{n}$, solutions of (5) and (6).

LEMMA 1. Let $T$ be an integral operator with a kernel $k$ of order l, of type i) or ii). If $f \in C_{\Delta}^{l}$ then, in both cases, $x, \tilde{x}_{n}$ and $\tilde{\phi}_{n}$ are in $C_{\Delta}^{l}$.

Now with $k_{t}(\cdot):=k(t, \cdot)$ for $t$ fixed in $[0,1]$, we consider the equation $\left(T^{*}-\bar{z}\right) l_{t}=k_{t}$, for $z \in \rho(T)$. 
LEMMA 2. When $k$ is a smooth kernel of order $l$, then $l_{t} \in C_{\Delta}^{l}(0,1)$ for any $t$ in $[0,1]$. When $k$ is a Green's kernel of order $l$ and continuity $\delta$, then $l_{t_{i}} \in C_{\Delta}^{l}(0,1)$ for $t_{i} \in \Delta, i=0, \ldots, n$, and $l_{t} \in C^{\delta}(0,1)$ for $t \notin \Delta$.

It is left to the reader to check the two lemmas (see Lebbar [10]). Note that when $k$ is a Green's kernel, $l_{t}$ is defined by the functions $l_{1} \in C^{\prime}(0, t), l_{2} \in$ $C^{\prime}(t, 1)$.

Lemma 2 shows that $l_{t}$ has the same smoothness properties as $k_{t}$. The same is true for $l_{t}^{\prime}$.

We define $\alpha:=\min (l, r+1)$ and $\alpha^{*}:=\min (l, r+1, \delta+2)$.

\section{1. $\Pi_{n}$ is an orthogonal projection}

THEOREM 3. Let $\Delta$ be quasi-uniform. With the above definitions, then for $f \in C_{\Delta}^{\prime}$, and $z$ in $\rho(T)$ :

i) if $k$ is a smooth kernel of order $l$, then for $t \in[0,1]:\left|\left\langle l_{t},\left(1-\Pi_{n}\right) f\right\rangle\right|<$ $C h^{2 \alpha}\left\|l_{t}^{(\alpha)}\right\|_{\Delta}$, and globally $\left\|T\left(1-\Pi_{n}\right) f\right\|_{\infty} \leqslant C h^{2 \alpha}$.

ii) if $k$ is a Green's kernel of order $l$ and continuity $\delta, 0 \leqslant \delta<l$, then for $t_{i} \in \Delta$,

$$
\left|\left\langle l_{t_{i}},\left(1-\Pi_{n}\right) f\right\rangle\right| \leqslant C h^{2 \alpha}\left\|l_{t_{i}}^{(\alpha)}\right\|_{\Delta}, \quad i=0, \ldots, n,
$$

for $t \notin \Delta$

$$
\left|\left\langle l_{t},\left(1-\Pi_{n}\right) f\right\rangle\right| \leqslant C h^{\alpha+\alpha^{*}} \max \left(\left\|l_{1 t}^{(\delta+1)}\right\|_{\infty},\left\|l_{2 i}^{(\delta+1)}\right\|_{\infty}\right),
$$

and globally, $\left\|T\left(1-\Pi_{n}\right) f\right\|_{\infty} \leqslant C h^{\alpha+\alpha^{*}}$.

Proof. It is adapted from Chandler [6]. Since $\Pi_{n}$ is an orthogonal projection: $\left\langle l_{t},\left(1-\Pi_{n}\right) f\right\rangle=\left\langle\left(1-\Pi_{n}\right) l_{t},\left(1-\Pi_{n}\right) f\right\rangle$. And

$$
\int_{0}^{1}\left(1-\Pi_{n}\right) l_{t}(s)\left(1-\Pi_{n}\right) f(s) d s=\sum_{i=1}^{n} \int_{\Delta_{t}}(1-\Pi) l_{i t}(s)(1-\Pi) f_{i}(s) d s .
$$

Given $f_{i} \in C_{\left(\Delta_{1}\right)}, \Pi f_{t}$ is the orthogonal projection of $f_{i}$ on the set of polynomials of degree $\leqslant r$ on $\Delta_{i}$. When $f, l_{t} \in C_{\Delta}^{l}(0,1), f_{i}, l_{i t} \in C_{\left(\Delta_{i}\right)}^{l}$, and

$$
\left\|(1-\Pi) f_{i}\right\|_{1} \leqslant C h^{\alpha+1}\left\|f_{i}^{(\alpha)}\right\|_{\infty},\left\|(1-\Pi) l_{i t}\right\|_{\infty} \leqslant C h^{\alpha}\left\|l_{i t}^{(\alpha)}\right\|_{\infty} .
$$

When $l_{t} \in C^{\delta}(0,1)$, with $t_{i-1}<t<t_{i}$, then on $\Delta_{i}$, if $\delta \leqslant r$,

$$
\left\|(1-\Pi) l_{i t}\right\|_{\infty} \leqslant C h^{\delta+1} \max \left(\left\|l_{i t}^{(\delta+1)}\right\|_{\infty},\left\|l_{2 i}^{(\delta+1)}\right\|_{\infty}\right) \text {. }
$$

The result follows by summing over $i$, and using $n h<\sigma$.

\section{2. $\Pi_{n}$ is an interpolatory projection}

Let $f$ be a function of $C^{l+1}(a, b)$, such that $f\left(w_{j}\right)=0, j=1, \ldots, r+1$, where the $\left\{w_{i}\right\}_{1}^{r+1}$ are $r+1$ distinct points in $(a, b)$. The $(r+1)$ th divided difference 
of $f$ on the points $w_{1}, \ldots, w_{r+1}$ is denoted by $\delta\left[w_{1}, w_{2}, \ldots, w_{r+1}, \cdot\right] f$. Then

$$
f(s)=\left(s-w_{1}\right) \cdots\left(s-w_{r+1}\right) \delta\left[w_{1}, w_{2}, \ldots, w_{r+1}, s\right] f, \text { for } s \notin\left\{w_{j}\right\}_{1}^{r+1} \text {. }
$$

We set, for $s \in[a, b]$,

$$
g^{*}(s)= \begin{cases}f(s) / v(s) & \text { if } s \notin\left\{w_{j}\right\}_{1}^{r+1}, \\ \lim _{t \rightarrow w_{j}}\left(\frac{f(s)}{v(s)}\right) & \text { if } t=w_{j}, j=1, \ldots, r+1,\end{cases}
$$

where $v(s)=\left(s-w_{1}\right) \cdots\left(s-w_{r+1}\right)$.

LeMmA 4. If $f \in C^{l+1}(a, b)$, then $g^{*} \in C^{l}(a, b)$.

There is only a need to prove that $g^{*}$ is $C^{l}$ in the neighborhood of any $w_{j}$, $j=1, \ldots, r+1$ (see Lebbar [10]). If $f \in C^{l+1}(a, b)$, the divided difference $\delta\left[w_{1}, \ldots, w_{r+1}, \cdot\right] f$ may therefore be prolongated by continuity on $[a, b]$, up to the order $l$.

We shall apply this lemma on each $\Delta_{i}$, with the $\left\{w_{j}\right\}_{1}^{r+1}$ being the Gauss points $\left\{\tau_{j}^{i}\right\}_{j=1}^{r+1}$. For $f \in C_{\Delta}^{l+1}, \Pi f_{i}$ is the polynomial of degree $<r$ on $\Delta_{i}$ which interpolates $f_{t}$ at the Gauss points $\left\{\tau_{j}^{i}\right\}_{j=1}^{r+1}$. Hence $(1-\Pi) f_{i}\left(\tau_{j}^{i}\right)=0$ for $j=$ $1, \ldots, r+1$. We consider the divided difference $\delta\left[\tau_{1}^{i}, \ldots, \tau_{r+1}^{i}, \cdot\right](1-\Pi) f_{i}$, and set $q_{i t}:=l_{i t} \delta\left[\tau_{1}^{i}, \ldots, \tau_{r+1}^{i}, \cdot\right](1-\Pi) f_{i} . k_{i t} \in C_{\left(\Delta_{1}\right)}^{l+1}$ (resp. $\left.C_{(\Delta,)}^{\delta}\right)$ implies that $l_{i t} \in C_{\left(\Delta_{i}\right)}^{l+1}\left(\right.$ resp. $\left.C_{\left(\Delta_{t}\right)}^{\delta}\right)$ and $q_{i t} \in C_{\left(\Delta_{i}\right)}^{l}$ (resp. $C_{\left(\Delta_{i}\right)}^{\delta}$, for $\delta \leqslant l$ ).

THEOREM 5. With the above definitions, then for $f \in C_{\Delta}^{l+1}$ and $z$ in $\rho(T)$

i) if $k$ is a smooth kernel of order $l$, then for $t \in[0,1],\left|\left\langle l_{t},\left(1-\Pi_{n}\right) f\right\rangle\right|<$ $C h^{r+1+\alpha}\left\|q_{t}^{(\alpha)}\right\|_{\Delta}$ and globally $\left\|T\left(1-\Pi_{n}\right) f\right\|_{\infty} \leqslant M_{\Delta} h^{r+1+\alpha}$,

ii) if $k$ is a Green's kernel, of order l and continuity $\delta, 0 \leqslant \delta<l$, then for $t_{i} \in \Delta$,

$$
\left|\left\langle l_{t_{i}},\left(1-\Pi_{n}\right) f\right\rangle\right| \leqslant C h^{r+1+\alpha}\left\|q_{t_{i}}^{(\alpha)}\right\|_{\Delta}, \quad i=0, \ldots, n,
$$

for $t \notin \Delta$,

$$
\left|\left\langle l_{t},\left(1-\Pi_{n}\right) f\right\rangle\right| \leqslant C h^{r+1+\alpha^{\bullet}} \max \left(\left\|q_{t}^{(\delta+1)}\right\|_{\infty},\left\|q_{2 t}^{\delta+1)}\right\|_{\infty}\right),
$$

and globally $\left\|T\left(1-\Pi_{n}\right) f\right\|_{\infty} \leqslant M_{\Delta} h^{r+1+\alpha^{*}}$.

If $f \in C_{\Delta}^{l+r+1}$, then $M_{\Delta} \leqslant C$.

Proof. It is adapted from de Boor-Swartz [1].

$$
\begin{aligned}
& \int_{0}^{1} l_{t}(s)\left(1-\Pi_{n}\right) f(s) d s=\sum_{i=1}^{n} \int_{\Delta_{i}} l_{t t}(s)(1-\Pi) f_{i}(s) d s \\
& =\sum_{i=1}^{n} \int_{\Delta_{i}} \underbrace{l_{i t}(s) \delta\left[\tau_{1}^{i}, \ldots, \tau_{r+1}^{i}, s\right](1-\Pi) f_{i}(s)}_{q_{i t}(s)} \underbrace{\left(s-\tau_{i}^{i}\right) \cdots\left(s-\tau_{r+1}^{i}\right) d s .}_{0(s)}
\end{aligned}
$$


When $q_{i t} \in C_{\left(\Delta_{j}\right)}^{l}, q_{i t}(s)=q_{i t}\left(t_{t-1}\right)+\cdots+\left(\left(s-t_{i-1}\right)^{l} / l !\right) q_{i t}^{(l)}\left(\theta_{s}\right), t_{i-1}<\theta_{s}<s$. Making use of $\int_{\Delta_{i}} v(s) p(s) d s=0$ for all polynomial $p$ of degree $<r$ on $\Delta_{i}$, we get $\left|\int_{\Delta_{i}} q_{i t}(s) v(s) d s\right| \leqslant C h^{r+2+\alpha}\left\|q_{i t}^{(\alpha)}\right\|_{\infty}$, which gives, for $l_{\ell} \in C_{\Delta}^{l}$,

$$
\left|\left\langle l_{t},\left(1-\Pi_{n}\right) f\right\rangle\right|\left\langle C h^{r+1+\alpha}\left\|q_{t}^{(\alpha)}\right\|_{\Delta} .\right.
$$

When $l_{i t} \in C_{(\Delta)}^{\delta}$,

$$
\left|\int_{\Delta_{t}} l_{i t}(s)(1-\Pi) f_{i}(s) d s\right| \leqslant C h^{r+2+\min (r+1, \delta+2)}
$$

and $\left|\left\langle l_{t},\left(1-\Pi_{n}\right) f\right\rangle\right| \leqslant C h^{r+1+\alpha^{*}}$, by summing over $i$.

Theorems 3 and 5 play a central role to derive the convergence rates, as we shall see in the next section.

\section{Convergence rates}

We recall that $\alpha=\min (l, r+1)$ and $\alpha^{*}=\min (l, r+1, \delta+2)$. In practice $\delta+2 \leqslant r+1 \leqslant l$, so that $\alpha=r+1$ and $\alpha^{*}=\delta+2$. We assume throughout this section that the kernel $k$ is of order $l$ for the Galerkin method $\left(f \in C_{\Delta}^{l} \Rightarrow \tilde{x}_{n}\right.$ $\left.\in C_{\Delta}^{l}, \tilde{\phi}_{n} \in C_{\Delta}^{l}\right)$ and of order $l+r+1$ for the collocation method $\left(f \in C_{\Delta}^{l+r+1}\right.$ $\Rightarrow \tilde{x}_{n} \in C_{\Delta}^{l+r+1}$ and $\tilde{\phi}_{n} \in C_{\Delta}^{l+r+1}$ ).

\subsection{Convergence rate for the eigenvalues}

The definitions are those of Section 2.2.

THEOREM 6. For both types of kernel $k$

$$
\lambda-\hat{\lambda}_{n}=O\left(\varepsilon_{n}\right), \quad \max _{i}\left|\lambda-\lambda_{i n}\right|=O\left(\varepsilon_{n}^{1 / \mu}\right), \quad \min _{i}\left|\lambda-\lambda_{i n}\right|=O\left(\varepsilon_{n}^{g / m}\right)
$$

where (a) $\varepsilon_{n}=h^{2 a}$ for the Galerkin method, and (b) $\varepsilon_{n}=h^{r+1+a}$ for the collocation method.

Proof. It is adapted from de Boor-Swartz [2] where it is noticed that $\lambda$ (resp. $\left.\lambda_{i n}\right)$ are the eigenvalues of two $m \times m$ matrices such that the $(i, j)$ th coefficient of the difference is $\left\langle x_{i}^{*},\left(1-\Pi_{n}\right) T\left(P_{I_{M_{n}}}\right)^{-1} x_{j}\right\rangle$. Theorem 3 applies where $l_{t}$ is replaced by $x_{i}^{*} \in C_{\Delta}^{l}$ and $T\left(P_{i_{M_{n}}}\right)^{-1} x_{j} \in C_{\Delta}^{l}$, if the kernel is of order $l$. Similarly, Theorem 5 applies if $k$ is of order $l+r+1$. And the results follow from classical theorems in matrix theory (see Wilkinson [18], pp. 80-81). 


\subsection{Convergence rate for the solutions and the eigenvectors}

(a) The Galerkin method. We suppose that $f \in C_{\Delta}^{l}$ and $k$ is of order $l$. Then $\tilde{x}_{n}$, $\tilde{\phi}_{n} \in C_{\Delta}^{l} . \Delta$ is quasi-uniform.

THEOREM 7. With a smooth kernel, $\left\|x-\tilde{x}_{n}\right\|_{\infty}$ and $\operatorname{dist}\left(\tilde{\phi}_{n}, M\right)$ are of the order $h^{2 \alpha}, \operatorname{dist}\left(\tilde{\phi}_{n}, E\right)=O\left(h^{2 \alpha / \mu}\right)$. With a Green's kernel, then: at $t_{i} \in \Delta,\left|x\left(t_{i}\right)-\tilde{x}_{n}\left(t_{i}\right)\right|$ and $\left|\tilde{\phi}_{n}\left(t_{i}\right)-\left(P \tilde{\phi}_{n}\right)\left(t_{i}\right)\right|$ are of the order $h^{2 \alpha}, i=0, \ldots, n$, whereas globally $\left\|x-\tilde{x}_{n}\right\|_{\infty}$ and $\operatorname{dist}\left(\tilde{\phi}_{n}, M\right)$ are of the order $h^{\alpha+\alpha^{*}}$, dist $\left(\tilde{\phi}_{n}, E\right)=O\left(h^{2 \alpha / \mu}\right)$, for $\mu>1$.

Proof. We apply Theorem 3 to $\left(\bar{x}_{n}-x\right)(t)=\left\langle l_{t},\left(1-\Pi_{n}\right) \tilde{x}_{n}\right\rangle,\left(\tilde{\phi}_{n}-P \tilde{\phi}_{n}\right)(t)$ $=\left\langle l_{i}^{\prime},\left(1-\Pi_{n}\right) \tilde{\phi}_{n}\right\rangle$, and Theorem 6 to

$$
\operatorname{dist}\left(\tilde{\phi}_{n}, E\right) \leqslant C\left(\left\|T\left(1-\Pi_{n}\right) \tilde{\phi}_{n}\right\|_{\infty}+\left|\lambda_{n}-\lambda\right|\right) .
$$

(b) The collocation method. We suppose that $f \in C_{\Delta}^{l+r+1}$ and $k$ is of order $l+r+1$. Then $\tilde{x}_{n}, \tilde{\phi}_{n} \in C_{\Delta}^{l+r+1}$. We get, as Theorem 8, the analog of Theorem 7 , where $h^{2 \alpha}$ (resp. $h^{\alpha+\alpha^{*}}$ ) is replaced by $h^{r+1+\alpha}\left(\right.$ resp. $\left.h^{r+1+\alpha^{*}}\right)$. The convergence rates in Theorems 7 and 8 are the best we could hope from the known results. It should be noticed that the computation of $\tilde{x}_{n}$ (resp. $\tilde{\phi}_{n}$ ) from $x_{n}$ (resp. $\lambda_{n}, \phi_{n}$ ) does not require much extra work: let $\operatorname{dim} X_{n}=n$ (say), let $\left\{e_{i}^{n}\right\}_{1}^{n}$ be a basis of $X_{n}$ : if $x_{n}=\sum_{i=1}^{n} \xi_{i}^{n} e_{i}^{n}$, then $T x_{n}=\sum_{i=1}^{n} \xi_{i}^{n} T e_{i}^{n}$ where the $\left\{T e_{i}^{n}\right\}_{1}^{n}$ have already been computed to get the coefficients of the matrix associated with the projection method.

\section{Numerical Example}

We end this paper with a numerical example illustrating the behavior of the iterated collocation solution for the Fredholm equation

$$
\int_{0}^{1} k(t, s) x(s) d s-\frac{1}{4} x(t)=-\cosh (1), \quad 0<t<1,
$$

with

$$
k(t, s)= \begin{cases}-t(1-s) & \text { if } s \geqslant t \\ -s(1-t) & \text { if } s<t .\end{cases}
$$

The exact solution is $x(t)=\cosh (2 t-1)$.

We choose the partition $\Delta=\{i / 5\}_{0}^{5}, h=\frac{1}{5}$, and on each interval $\Delta_{i}$, the $r+1=4$ Gauss points. We display in Table 1 the values of $x-x_{n}$ and $x-\tilde{x}_{n}$ 
at the partition points $t_{i}, i=1,2,3,4$. The kernel $k$ is of "Green's function" type with $\delta=0$.

TABLE 1

Error values at the partition points

\begin{tabular}{c|c|c|c}
$i$ & $\left(x-x_{n}\right)\left(t_{i}^{-}\right)$ & $\left(x-x_{n}\right)\left(t_{i}^{+}\right)$ & $\left(x-\tilde{x}_{n}\right)\left(t_{i}\right)$ \\
\hline 1 & $8.10^{-1}$ & $7.10^{-5}$ & $-5.10^{-12}$ \\
2 & $6.10^{-5}$ & $6.10^{-5}$ & $-7.10^{-12}$ \\
3 & $6.10^{-5}$ & $6.10^{-5}$ & $-7.10^{-12}$ \\
4 & $7.10^{-5}$ & $8.10^{-5}$ & $-5.10^{-12}$ \\
\hline
\end{tabular}

\section{References}

[1] C. de Boor and B. Swartz, "Collocation at Gaussian points", SIAM J. Num. Anal. 10 (1973), 582-606.

[2] C. de Boor and B. Swartz, "Collocation approximation to eigenvalues of an ordinary differential equation: the principle of the thing", Math. Comp. 35 (1980), 679-694.

[3] C. de Boor and B. Swartz, "Collocation approximation to eigenvalues of an ordinary differential equation: numerical illustrations", Math. Comp. 36 (1981).

[4] C. de Boor and B. Swartz, "Local piecewise polynomial projection methods for an ode which give high-order convergence at knots", Math. Comp. 36 (1981).

[5] G. A. Chandler, "Global superconvergence of iterated Galerkin solutions for second kind integral equations", Australian National University Technical Report (1978).

[6] G. A. Chandler, Superconvergence of numerical solutions of second kind integral equations ( $\mathrm{Ph}$. D. Thesis, Australian National University, 1979).

[7] F. Chatelin, "Numerical computation of the eigenelements of linear integral operators by iterations", SIAM J. Num. Anal. 15 (1978), 1112-1124.

[8] F. Chatelin, "Sur les bornes d'erreur a posteriori pour les éléments propres d'opérateurs linéaires", Numer. Math. 32 (1979), 233-246.

[9] F. Chatelin, Linear spectral approximation in Banach spaces (Academic Press, New-York, to appear).

[10] R. Lebbar, "Méthode de Galerkin iterée pour l'équation intégrale de Fredholm de $2 e$ espèce" (Rapport D. E. A., Université de Grenoble, 1980).

[11] Lin Qun and Liu Jiaquan, "Extrapolation methods for Fredholm integral equations with non-smooth kernels" Numer. Math. 35 (1980), 459-464.

[12] G. R. Richter, "Superconvergence of piecewise polynomial Galerkin approximations for Fredholm integral equations of the 2nd kind", Numer. Math. 31 (1978), 63-70.

[13] I. H. Sloan, "Error analysis for a class of degenerate kernel methods", Numer. Math. 25 (1976), 231-238.

[14] I. H. Sloan, "Iterated Galerkin method for eigenvalue problems", SIAM J. Num. Anal. 13 (1976), 753-760.

[15] I. H. Sloan, "A review of numerical methods for Fredholm equations of the 2nd kind", in the Application and numerical solution of integral equations (eds. Anderssen, de Hoog, Lukas, Sijthoff \& Noordhoff, Leyden, 1980). 
[16] I. H. Sloan and B. J. Burn, "Collocation with polynomials for integral equations of the 2nd kind: a new approach to the theory", Journ. Integral Equ. 1 (1979), 77-94.

[17] I. H. Sloan, E. Noussair and B. J. Burn, "Projection method for equations of the 2nd kind" $J$. Math. Anal. Appl. 69 (1979), 84-103.

[18] J. H. Wilkinson, The algebraic eigenvalue problem (Clarendon Press, Oxford, 1965).

Université de Grenoble

Mathématiques Appliquées, IMAG

BP 53X, 38041 GRENOBLE-CEDEX

France 